\title{
Attenuation of Cardiomyopathy Induced in Sub-Chronic Exposure of Acrolein by Sulforaphane via Indirect PPARy Expression Promoter
}

\author{
Promise Madu Emeka ${ }^{1, *}$, Hairul-Islam Mohamed Ibrahim², Mohamed Aly Morsy ${ }^{1,3}$, \\ Ibrahim Abdulraham Alhaider ${ }^{1,4}$, Snawar Hussian ${ }^{5}$, Emad Abdelaziz Ahmed ${ }^{2,6}$ \\ ${ }^{1}$ Department of Pharmaceutical Sciences, College of Clinical Pharmacy, King Faisal University, Al-Ahsa, SAUDI ARABIA. \\ ${ }^{2}$ Department of Biological Sciences, College of Science, King Faisal University, Al-Ahsa, SAUDI ARABIA. \\ ${ }^{3}$ Department of Pharmacology, Faculty of Medicine, Minia University, El-Minia, EGYPT. \\ ${ }^{4} R \& D$, Saudi Food and Drug Authority, Riyadh, SAUDI ARABIA. \\ ${ }^{5}$ Department of Biomedical Sciences, College of Clinical Pharmacy, King Faisal University, Al-Ahsa, SAUDI ARABIA. \\ ${ }^{6}$ Lab of Molecular Physiology, Department of Zoology, Faculty of Science, Assiut University, Assiut, EGYPT.
}

\begin{abstract}
Sulforaphane (SPN) is reported to activate the Nrf2/Keap1 complex responsible for protein and gene expression promotion of various antioxidant enzymes. The present study examined the role of $\mathrm{Nrf2}$ in modulating other signaling pathways involved in SPN's attenuation of acrolein (ACL)-induced cardiomyopathy in rats. Forty-two rat was categorized into seven 4-week treatment groups: control, SPN, losartan (LTN), $A C L, A C L+S P N, A C L+L T N$, and $A C L+S P N+L T N$. Heart samples were harvested for analysis; cardiac oxidative and injury biomarker levels and histopathological examination were undertaken. PPAR $\gamma, \mathrm{Nrf2}$, NF- $\mathrm{KB}, \mathrm{COX}-2$, and CYP2E1 protein expressions were examined. Results show that SPN and SPN + LTN reduced GSH, catalase, and lipid peroxidation compared to the ACL-treated group. Also, levels of creatine kinase-MB, cardiac troponin, and caspase 3 induced by $A C L$ were all attenuated. Altered cardiac tissue pathophysiology by ACL was alleviated. SPN + LTN significantly increased Nrf2 expression via PPAR $\gamma$ action but decreased NF- $\mathrm{B}$ B and COX-2 expressions. Also, ACLincreased CYP2E1 expression was significantly attenuated by the SPN + LTN combination. For the first time, it suggests that SPN + LTN might offer a better therapeutic alternative to ACL-induced cardiomyopathy by activating Nrf2 via PPAR $\gamma$ and reducing NF- $\mathrm{B} / \mathrm{COX}$ 2/CYP2E1 expressions.
\end{abstract}

Key words: Sulforaphane, Losartan, Acrolein, Cardiomyopathy, Oxidative Stress, PPAR $;$ Nrf2.

\section{INTRODUCTION}

Acrolein (ACL) is an environmental pollutant and dietary aldehyde found in high concentrations in foods like cheese, donuts, and coffee. ${ }^{1,2}$ It is implicated in causing chronic cardiac disease, among other organ effects, by reacting with nucleophiles such as DNA and protein molecules to cause oxidative stress and myocardial damage. ${ }^{3,4}$ The heart is usually a known target for assault by numerous agents, including ACL, because it is one of the most energy-demanding organs. In this regard, cardiomyopathy becomes the likely outcome of most drugs' assault on the heart leading to failure. The mechanism of drug-induced cardiomyopathy is varied but related mostly to altered myocardial energy homeostasis and metabolic pathways. The heart function is regulated by multicomplex signaling protein pathways that control its activity. ${ }^{5}$ Exposure to ACL can exacerbate inflammatory activity by promoting mediators and prooxidant activities, causing oxidative stress that can lead to cardiomyopathy. ${ }^{3,6}$ Accumulated evidence indicates that it can change the expressions of protein signaling moieties particularly that of nuclear factor
Submission Date: 14-10-2021; Revision Date: 01-11-2021; Accepted Date: 22-11-2021

DOI: 10.5530/ijper.55.4.205 Correspondence: Dr. Promise Madu Emeka Department of Pharmaceutical Sciences, College of Clinical Pharmacy, King Faisal University, Al-Ahsa 31982, SAUDI ARABIA.

Phone no: +966503239033

E-mail: pemeka@kfu.edu.sa

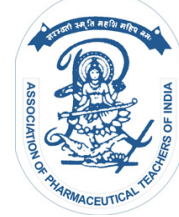

www.ijper.org 
erythroid 2-related factor 2 (Nrf2) transcription factor and peroxisome proliferator-activated receptor (PPAR) superfamily. ${ }^{7}$ These signaling proteins are reported to play essential roles in adapting to homeostatic changes by modifying the expressional transcriptions of chief antioxidant enzymes, ${ }^{8}$ particularly enzymes involved in protein-gene expression and antioxidant enzyme actions. ${ }^{5,910}$ Of note is that PPARy's interactional activities on the heart are still controversial in terms of their inverse relationships yet to be distinctly clear. ${ }^{11}$ However, studies have shown that inhibition of PPAR $\gamma$ activities in the cardiomyocytes, either induced by drug or diseased conditions, will promote inflammatory events of mediators. A consequent oxidative stress situation will ensue, leading to cardiomyopathy. ${ }^{12}$ Therefore, it could be interpreted to mean that increased expression of PPAR $\gamma$ on the heart will likely nullify the generation of pro-inflammatory mediators. Another nuclear transcription factor described as an antioxidant response element, Nrf2, has been documented to protect against inflammation and oxidative injury; it has been reported to induce the expression of PPAR $\gamma{ }^{13,14}$ Together, both play a critical protective role during inflammation and oxidative stress-induced cellular injury. Hence, studies have demonstrated a reciprocal transcriptional regulation between genes of $\mathrm{Nrf2}$ and PPAR $\gamma \cdot{ }^{13}$ Evidence has shown that sulforaphane (SPN) (a dietary isothiocyanate) acts by inducing Nrf2 signaling, promoting an antioxidant environment that alleviates inflammation and tissue oxidative stress ${ }^{15,16}$ From the foregoing, SPN, if supplemented with PPAR $\gamma$ agonist, might be an effective therapeutic combination in attenuating inflammatory and oxidative stress induced by ACL. SPN is a natural sulfur compound derived from glucosinolate found in cruciferous vegetables like cabbages, cauliflower, broccoli, and olives. ${ }^{15,17} \mathrm{We}$, therefore, hypothesized that the PPAR $\gamma$ expression ratio on the heart might be decreased in inflammatory and oxidative stress conditions induced by ACL. Hence it might be beneficial to use PPAR $\gamma$ agonist in this regard. This is because these regulatory proteins are very sensitive to such environments. In addition, reduced cardiac expressions of these signaling proteins may increase the risk of assault, as its functionality may be compromised. We also hypothesize that Nrf2-mediated protein expression of PPAR $\gamma$ might be enhanced preferentially, thus contribute to the attenuation of the development of ACL-induced cardiomyopathy. The aim of the present study was to evaluate if ACL can modulate the expressions of Nrf2 and PPARy by promoting the expressions of nuclear factor kappalight-chain-enhancer of activated B cells (NF-xB), cyclooxygenase-2 (COX-2), and cytochrome P450 2E1 (CYP2E1) in cardiomyopathy state and investigate the effect of losartan (LTN), a PPARy agonist, on Nrf2 activator SPN in ACL-induced cardiomyopathy and their effect on antioxidant activities. To date, there is no work done to evaluate the effect of SPN plus LTN on ACLinduced cardiomyopathy. This study will confirm the extent of their involvement and the interplay of these signaling pathways in ACL-induced cardiomyopathy. Also, it will adduce a more beneficial treatment that will improve the outcome of this disease among vulnerable patients.

\section{MATERIALS AND METHODS}

\section{Chemicals, Assay kits, and Antibodies}

SPN, LTN, and ACL were purchased from SigmaAldrich (St. Louis, MO, USA). Assay kits for reduced glutathione (GSH) were procured from Sigma-Aldrich, and for catalase, lipid peroxidation, and caspase 3 were purchased from BioVision (Milpitas, CA, USA). Creatine kinase-MB (CK-MB) and cardiac troponin were from MyBioSource (San Diego, CA, USA). Primary antibodies against PPAR $\gamma, \mathrm{Nrf2}$, and NF- $x \mathrm{~B}$ (p65) were purchased from Santa Cruz Biotechnology (Dallas, TX, USA), while COX-2 and CYP2E1 were from Abcam (Waltham, MA, USA). Gels for Western blotting were procured from Bio-Rad (Hercules, CA, USA).

\section{Animals}

A total of 42 Wistar rats (males and females) with a weight range of 150-200 $\mathrm{g}$ were used for this study. They were obtained from the Animal House of College of Medicine, King Faisal University, Saudi Arabia. Rats were maintained in groups of six per cage in controlled environmental conditions according to the specified standards, with $12 \mathrm{hr}$ dark and $12 \mathrm{hr}$ light cycle, at $23 \pm 1{ }^{\circ} \mathrm{C}$. They were allowed access to food and water throughout the study. Animal care and experimental procedures were carried out according to the guidelines of the Research Ethics Committee at King Faisal University (KFU-REC/2021-06-07) and that of the National Committee of Bioethics (NCBE), King Abdulaziz City for Science and Technology (KACST), Saudi Arabia.

\section{Experimental Protocol}

The rats were divided into seven groups of six animals each and treated once a day for 4 weeks. ACL and SPN were suspended in $0.3 \% \mathrm{w} / \mathrm{v}$ methyl cellulose and given orally. Group I (control) received normal saline + $0.3 \% \mathrm{w} / \mathrm{v}$ methyl cellulose as a vehicle. Group II was treated with SPN $(1 \mathrm{mg} / \mathrm{kg}) .{ }^{18}$ Group III was given 
$10 \mathrm{mg} / \mathrm{kg}$ of PPAR $\gamma$ agonist (LTN).${ }^{19}$ Group IV animals were treated with ACL $5 \mathrm{mg} / \mathrm{kg} .{ }^{1}$ Group V was treated with SPN, and after $30 \mathrm{~min}$ it was given ACL. Group VI received LTN 30 min later was treated with ACL. Group VII was given SPN plus LTN, and after $30 \mathrm{~min}$ it was administered with ACL. After 4 weeks of treatment, animals were euthanized by cervical dislocation under ether anesthesia. The blood and heart samples were harvested at the same time. The heart samples were thoroughly rinsed in phosphate-buffered saline to remove excess blood before being stored at $-85^{\circ} \mathrm{C}$ for analysis. The collected samples were used to estimate biochemical markers of cardiac antioxidants, namely GSH, catalase, and lipid peroxidation. Also, cardiac injury markers, CK-MB, cardiac troponin, and caspase 3 were measured. Western blotting was performed to analyze the protein expressions of PPAR $\gamma, \mathrm{Nrf} 2, \mathrm{NF}-x \mathrm{~B}$, COX-2, and CYP2E1.

\section{Cardiac Oxidative Stress Evaluation}

Harvested heart samples were homogenized in phosphate-buffered saline in a ratio of $1: 10(\mathrm{w} / \mathrm{v})$ homogenates and then centrifuged at $13000 \mathrm{~g}\left(4^{\circ} \mathrm{C}\right)$ for $30 \mathrm{~min}$; the supernatant was then collected for assay. GSH levels were determined following the instructions of the manufacturer. Briefly, it is a colorimetric reaction of 5,5'-dithiobis(2-nitrobenzoic acid). Cardiac GSH contents were measured at $412 \mathrm{~nm}$ using a standard curve plot. Catalase activity was determined spectrophotometrically using a commercial kit according to the manufacturer's instructions. Essentially, in the assay, catalase first reacts with $\mathrm{H}_{2} \mathrm{O}_{2}$ to produce water and oxygen, the unconverted $\mathrm{H}_{2} \mathrm{O}_{2}$ reacts with OxiRed ${ }^{\mathrm{TM}}$ probe to produce a product, which is then measured at $570 \mathrm{~nm}$. Concentrations of MDA, as a marker for lipid peroxidation, were determined by the thiobarbituric acid-reactive substances method by determining the levels of thiobarbituric acid-reactive substances, as earlier described by Emeka et al. ${ }^{20}$ Phosphoric acid solution $(1 \%)$ and a known volume of thiobarbituric acid were added to each homogenate sample and then incubated at $95^{\circ} \mathrm{C}$ for $1 \mathrm{hr}$. The concentration of the subsequent thiobarbituric acid-reactive substances was then measured spectrophotometrically at $535 \mathrm{~nm}$.

\section{Cardiac Injury Biomarkers}

CK-MB activity was evaluated according to manufacturers' procedure as described by Emeka et al..$^{20}$ Heart homogenates samples were added to the micro-ELISA plate wells treated with a specific antibody and incubated for $90 \mathrm{~min}$ at $37^{\circ} \mathrm{C}$. The liquid was decanted, and the biotinylated detection antibody specific for Rat CK-MB, and Avidin-Horseradish Peroxidase (HRP) conjugate were also added separately and incubated for another $1 \mathrm{hr}$ at the same temperature. Thereafter, substrate solution was added to the sample solutions, and enzyme reactions stopped by adding a stop solution. The ensuing colorful samples are then measured spectrophotometrically at a wavelength of $450 \mathrm{~nm}$. Cardiac troponin levels were assayed in heart samples by ELISA method following the kit manufacturer's instructions. Briefly, using purified rat cardiac troponin antibody, microtiter plates were coated with troponin antibody; then add HRP-Conjugate Reagent to form an antibody-antigenantibody-enzyme complex. The resultant solutions were washed several times, and a tetramethylbenzidine substrate solution was added. The ensuing reactions were terminated by the addition of sulfuric acid solution. The color developed is measured spectrophotometrically at a wavelength of $450 \mathrm{~nm}$.

\section{Cardiac Caspase 3 Activity Assay}

Caspase 3 activity was measured in the different treatment groups using colorimetric assay, according to the manufacturer's instructions as described by Emeka et $a .^{20}$ The heart tissues were homogenized in $3 \mathrm{ml}$ of $10 \mathrm{mM}$ phosphate buffer ( $\mathrm{pH}$ 7.4). Detection of caspase 3 activity was predicated on the hydrolysis of the peptide substrate acetyl-Asp-Glu-Val-Asp p-nitroanilide by caspase 3 . The reaction led to the consequent release of the p-nitroaniline component, which was then measured spectrophotometrically at $405 \mathrm{~nm}$.

\section{Histopathological Examination}

The hearts tissues were fixed in 10\% buffered formalin for at least $24 \mathrm{hr}$ and then processed using standard protocol for microscopical examination. Embedded paraffin sections were stained with hematoxylin and eosin. Slides were evaluated under a light microscope for pathology. Pathophysiological scores were used to evaluate levels of cardiac injury according to the modified grading method described by Billingham et al..$^{21}$ The control group was used to compare pathology observed in the different treatment groups as follows: $0=$ no pathology, $1=$ mild pathology, $2=$ moderate pathology, 3 = severe pathology.

\section{Protein Expression Determination Using Western Blotting Technique}

The protein expressions of PPAR $\gamma, \mathrm{Nrf} 2, \mathrm{NF}-\varkappa \mathrm{B}, \mathrm{COX}-$ 2 , and CYP2E1 in the heart tissues were determined through Western blotting using their specific antibodies, according to the method described earlier by Emeka et al. ${ }^{20}$ From the homogenized harvested heart samples treated with with radioimmunoprecipitation assay using lysis 
buffer, the nuclear and cytoplasmic extract were isolated and prepared. The isolated nuclear extracts were used for the determination of PPAR $\gamma, \mathrm{Nrf2}, \mathrm{NF}-x \mathrm{~B}$. Whereas the protein extracts were used to determine COX-2, and CYP2E1 expressions. Approximately $50 \mu \mathrm{g}$ of protein samples were separated using 10\% sodium dodecyl sulfate-polyacrylamide gel electrophoresis (SDS-PAGE) gels. These were later transferred onto polyvinylidene fluoride membranes, and 5\% non-fat dried milk was used to block the non-specific sites at $20-22^{\circ} \mathrm{C}$ for $1 \mathrm{hr}$. This was followed by washing the membranes with Tris-buffered saline and 0.1\% Tween 20 (TBST). The membranes were then incubated overnight at $4^{\circ} \mathrm{C}$ with primary mouse monoclonal antibodies against $\operatorname{PPAR} \gamma$, Nrf2, NF- $x \mathrm{~B}, \mathrm{COX}-2$, and CYP2E1 in a ratio of 1:1000. Thereafter, the membranes were washed with TBST and incubated with secondary peroxidase-conjugated goat anti-mouse $\mathrm{IgG}$ in a ratio of 1:5000 for $1 \mathrm{~h}$. The resulting bands were visualized according to the manufacturer's instructions, using an enhanced chemiluminescence system. ImageJ software (freeware; rsbweb.nih.gov/ij) was used to perform the densitometric analyses of the bands.

\section{Statistical Analysis}

Data obtained were expressed as mean $\pm \mathrm{SD}$, and analysis was done using GraphPad Prism software version 8.2 (San Diego, CA, USA). Comparison between control and treatment groups were made using the one-way analysis of variance, and the differences between the groups were measured using Tukey's multiple comparisons test. Statistically significant was taken as $p<0.05$.

\section{RESULTS}

\section{Cardiac Oxidative Biomarkers}

Figure 1 (A-C) represents graphs of cardiac oxidative biomarkers obtained from different experimental groups. A, represents GSH levels measured in heart samples after 4 weeks of treatments. Our data showed that ACL markedly $(p<0.0001)$ increased GSH levels $(39.06 \pm 1.2 \mathrm{nmol} / \mathrm{mg}$ protein) compared to control $(5.3 \pm 0.71 \mathrm{nmol} / \mathrm{mg}$ protein), indicating myocardial tissue adaptation to intense oxidative stress generated by ACL. However, pretreatment with SPN and LTN each reduced GSH levels significantly $(p<0.001)$ too to 18.8 \pm 0.92 and $20.9 \pm 0.95 \mathrm{nmol} / \mathrm{mg}$ protein, respectively, compared to ACL treatment. Moreover, combinations of SPN+LTN reduced the level further to $12.5 \pm 1.49$ $\mathrm{nmol} / \mathrm{mg}$ protein, significantly too $(\phi<0.001)$ compared to ACL treatment. B, represents heart sample catalase levels observed from different treatment groups. It
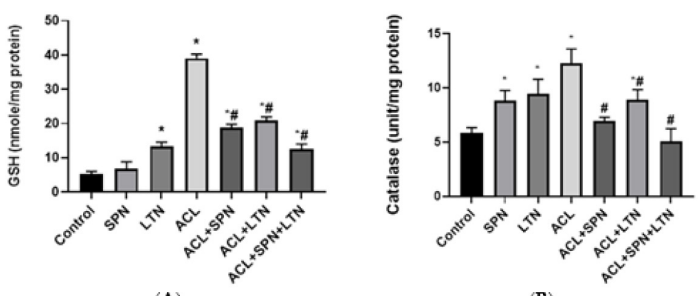

(A)

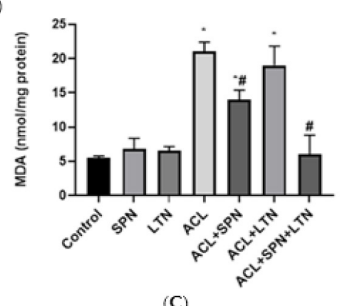

Figure 1: Effects of sulforaphane (SPN), losartan (LTN), and acrolein (ACL), and pre-treatments with SPN, LTN, and SPN+LTN on antioxidant enzymes activities after 4 weeks of treatment. A, reduced glutathione (GSH) levels with different treatment groups; B, Catalase activity; C, Lipid peroxidation showing levels of malondialdehyde (MDA). Results represent mean \pm SD. * Shows a significant difference $(p<0.05)$ compared to control; \# represents a significant difference between ACL and other pre-treatment groups.

showed that catalase activity was significantly increased $(\phi<0.01)$ by ACL compared to control $(12.3 \pm 1.34$ for ACL and $5.85 \pm 0.5 \mathrm{unit} / \mathrm{mg}$ protein for control). Our result also showed that pretreatment with a combination of SPN+LTN restored catalase activities significantly $(p<0.001)$ to $5.45 \pm 1.2 \mathrm{unit} / \mathrm{mg}$ protein compared to ACL treatments. In Figure 1C, we observed that ACL gave a significant increase $(p<0.001)$ in lipid peroxidation $(21.0 \pm 1.4 \mathrm{nmol} / \mathrm{mg}$ protein) compared to control $(5.5 \pm 0.3 \mathrm{nmol} / \mathrm{mg}$ protein). Results obtained also indicated that only the pretreatment with SPN diminished lipid peroxidation significantly $(\phi<0.05)$ compared to the ACL treatment given $14.0 \pm 1.4 \mathrm{nmol} / \mathrm{mg}$ protein compared to LTN pretreatment of $19.1 \pm 2.8 \mathrm{nmol} / \mathrm{mg}$ protein. However, the use of both agents brought lipid peroxidation to control levels. These results suggest that pre-treatment with a combination of SPN and LTN has better potential in abolishing ACL-induced oxidative stress and inflammatory changes. Hence preventing progressive cardiomyopathy, evidenced by diminished lipid peroxidation.

\section{Cardiac Injury Biomarkers}

Results presented in Figure 2 (A-C) are measurements of cardiac injury biomarker levels obtained from each experimental group. A, exhibits CK-MB levels of all the treatments carried out in this study. It showed a very significant increase $(p<0.001)$ in CK levels for the ACL group $(77.0 \pm 5.7 \mathrm{nmol} / \mathrm{mm} / \mathrm{mg})$. However, 


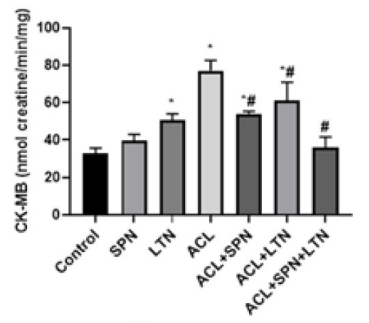

(A)

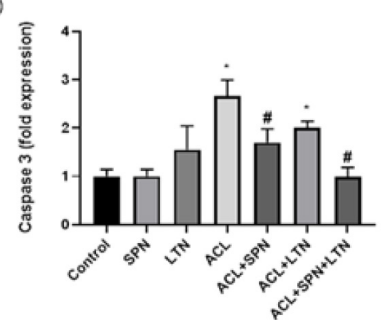

(C)

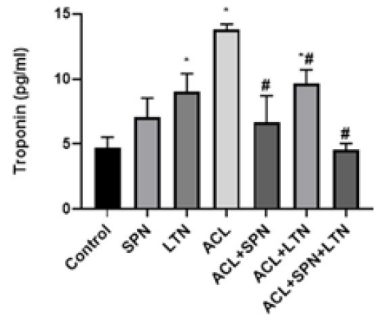

(B)

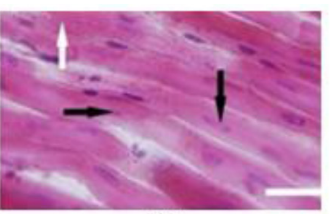

(A)

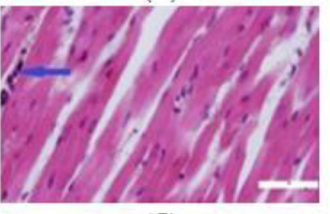

(C)

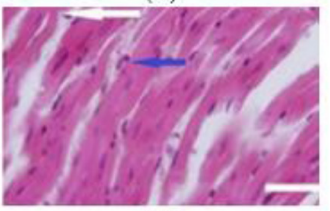

(E)

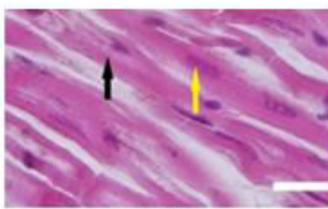

(B)

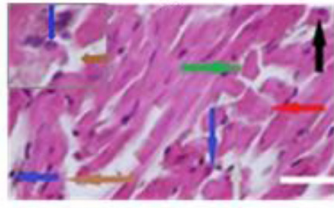

(D)

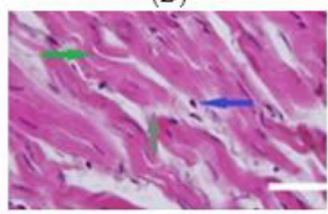

(F)
Figure 2: Effects of sulforaphane (SPN), losartan (LTN), and acrolein (ACL), and pre-treatments with SPN, LTN, and SPN+LTN on myocardial injury makers. A, Creatine kinaseMB (CK-MB); B, Cardiac troponin; C, Caspase 3 activities. Results represent mean \pm SD. * Shows a significant difference $(p<0.05)$ compared to control; \# represents a significant difference between ACL and other pre-treatment groups.

all the pretreatment groups of SPN, LTN, and their combination ameliorated ACL effects significantly $(p<0.001)$. In addition, the treatment combination restored CK level as seen with the control $(36.0 \pm 5.7$ and $33.0 \pm 2.8$ respectively). $\mathrm{B}$, represents cardiac troponin levels obtained from each experimental group. Compared to control $(4.70 \pm 0.85 \mathrm{pg} / \mathrm{ml})$, ACL produced a significant increase with $13.8 \pm 0.42 \mathrm{pg} / \mathrm{ml}$. We also found that both SPN and LTN pre-treatments reduced the effects of ACL-generated increase to $6.65 \pm 2.05$ and $9.6 \pm 1.06 \mathrm{pg} / \mathrm{ml}$, respectively. Furthermore, pre-treatment combination with SPN and LTN restored cardiac troponin level as seen with the control group $(4.55 \pm 0.49 \mathrm{pg} / \mathrm{ml})$. C, shows caspase 3 activity as determined in all experimental groups. Our data revealed a significant $(p<0.001)$ caspase 3 activity $(2.67 \pm 0.33)$ in rats treated with ACL compared to control $(1.1 \pm 0.14)$. On the other hand, SPN pre-treatment significantly $(\phi<0.01)$ reduced caspase 3 activity to $1.70 \pm 0.28$. Our results further indicated that the combination of SPN and LTN showed a better ameliorative effect on ACL-induced cardiomyopathy than when they were administered individually.

\section{Histopathological Examinations of Representative Heart Samples}

Figure 3 (A-G) illustrates the histopathology profile observed in each experimental group after 4 weeks of treatment. A, represents the control experimental group,

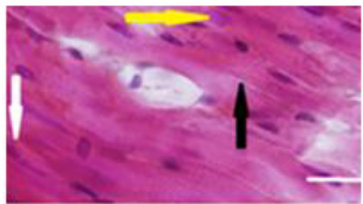

(G)

Figure 3: Histopathological analysis of heart samples after 4 weeks of treatments with sulforaphane (SPN), losartan (LTN), and acrolein (ACL), and pretreatments with SPN, LTN, and SPN+LTN (A-G; H\&E $\times 20$; scale bar $10 \mu \mathrm{m}$ ). A, Longitudinal section in control group showing a normal appearance, normal striations (white arrow) with a proper intercalated disc (black arrows); B, SPN-treated heart tissue showing normal monocyte nucleus (yellow arrow) and normal intercalated disc (black arrow); C, Cardiac muscle treated with LTN showing infiltration of neutrophils (blue arrow); D, ACL-treated cardiac muscle showing infiltration of cells (blue arrows), distorted striation (red arrow), interstitial fibrosis (brown arrow), wavy myocardial fibers (green arrow), and myofibril disarray arrow) and interstitial fibrosis (brown arrow); E, Cardiac tissue treated with $A C L+S P N$ showing distorted striation (white arrow) and infiltration of cells (blue arrow); F, ACL+LTN-treated cardiac tissue showing inflammation infiltration of neutrophils (blue arrow), wavy myocardial fibers (green arrow), and myofibril degeneration (grey arrow); G, SPN+LTN-treated cardiac tissue showing normal histoarchitecture of the heart tissue with normal striation (white arrow), monocyte nucleus (yellow arrow), and intercalated disc (black arrows).

with no apparent pathological changes of histoarchitecture, showing normal striation and intercalated disc. B, represents a photomicrograph of representative heart sample treated with SPN alone with no apparent histopathology visible. It shows a normal intercalated disc and monocyte nucleus. C, describes pathological features observed with LTN treatment alone, showing normal cardiac histoarchitecture with mild cells infiltration, confirming its antioxidant profile. $\mathrm{D}$, shows a representative photomicrograph of heart sample treated with ACL. It showed myocardial disarray, interstitial (black arrow). The insert $(\times 20)$ shows infiltration of cells (blue 
fibrosis, distorted intercalated disc, distorted striation, and in some cases, wavy myocardial fibers. These pathological features clearly indicate cardiomyopathy and correlate with observed cardiac injury biomarkers in ACL-treated rats. E, shows a representative of SPN pre-treated heart tissue, indicating mildly distorted striation and inflammatory cell infiltration. F, illustrates representative heart sample pre-treated with LTN before ACL administration. It shows recovery with mild wavy myocardial fiber with much reduced myocardial disarray and mild infiltrated inflammatory cells. Finally, $\mathrm{G}$, shows a representative heart sample pre-treated with a combination of SPN and LTN displaying normal monocyte nucleus, striation, and intercalated disc. This observation suggests complete inhibition of ACLinduced cardiac effects.

\section{Heart Tissue Histopathological Score Heatmap}

Figure 4 is the heatmap showing pathological scores for each myocardial change observed in the different experimental groups using Billingham's scoring method as aforementioned in the methodology. The heart sample from the control group had a score of zero (0) in all listed pathology because no pathology was observed. However, the heatmap indicated that the ACL-treated group had scores ranging from 1-3 in all pathology described, with the highest score of 3 for myocardial disarray, interstitial fibrosis, and distorted intercalated

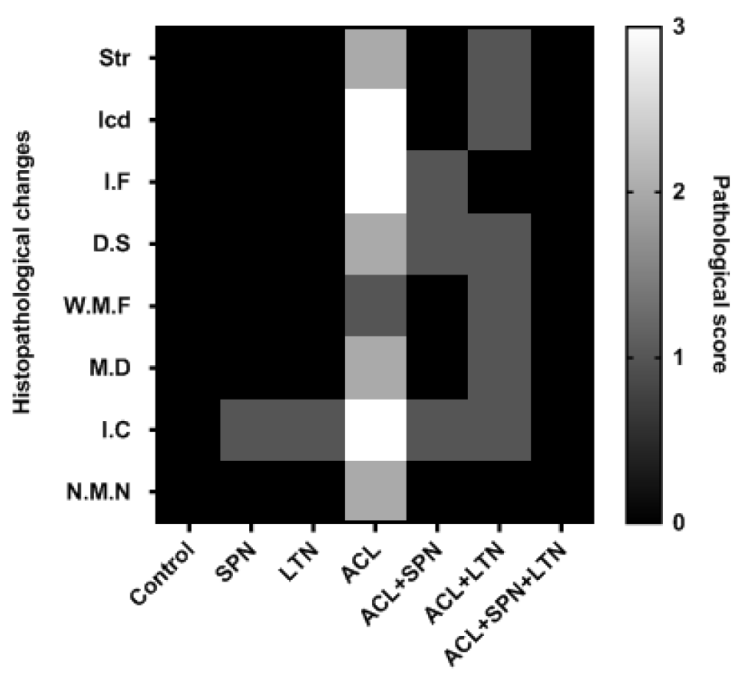

Figure 4: Heatmap evaluation score of cardiac histopathological changes induced by sulforaphane (SPN), losartan (LTN), and acrolein (ACL), and pretreatments with SPN, LTN, and SPN+LTN after 4 weeks. Histopathology score: $0=$ no pathology, 1 = mild pathology, 2 = moderate pathology, and 3 = severe pathology. Str = striation; Icd = intercalated disc; I.F = interstitial fibrosis; D.S = distorted striation; W.M.F = wavy myocardial fibers; M.D = myofiber disarray; I.C = infiltration of cells; N.M.N = normal monocyte nucleus. disc confirming cardiomyopathy. Pre-treatment with either LTN or SPN had a total of 1, indicating mild pathology. Pre-treatment with LTN had a higher combined score of 1 for each parameter except in interstitial fibrosis and myocardial disarray, compared to SPN, suggesting that SPN pre-treatment had a better profile of protection. Besides, pre-treatment with SPN+LTN showed complete protection from the adverse effects of ACL.

\section{Western Blotting Results}

The results of the Western blotting analysis are presented in Figure 5 (A-C). A, shows different bands of phosphorylated protein expressions in each experimental group. Our data showed in A, that ACL increased the level of Nrf2 compared to control. This means that the tissue was experiencing stress, and therefore the increase in $\mathrm{Nrf} 2$ can be described as oxidative stress adaptive measure. Pre-treatment with either LTN or SPN decreased this stress, as evidenced from histopathology observation. It was, however, noted that using SPN or LTN alone increased the levels of Nrf2 considerably compared to control, confirming the ability of SPN to induce Nrf2 protein expression. Interestingly, pre-treatment with SPN+LTN produced a profound increase in the expression of Nrf2 significantly $(p<0.0001)$ compared to control. Based on the overall results obtained, we suggest that

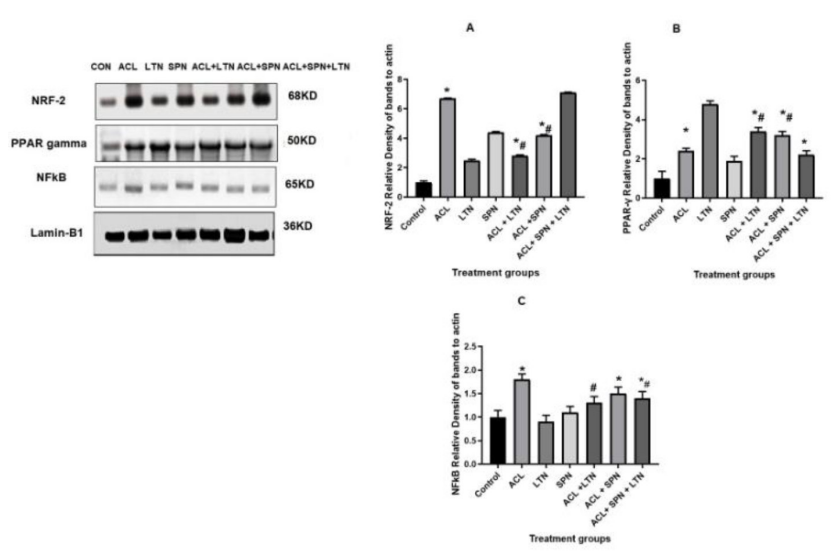

Figure 5: Effects of acrolein (ACL), losartan (LTN), and sulforaphane (SPN), and pretreatments with LTN, SPN, and SPN+LTN after 4 weeks on A, different bands of protein expressions, and relative density of laminin-B. A,nuclear factor erythroid 2-related factor 2 (NRF-2); B, peroxisome proliferator-activated receptor- $\gamma$ (PPAR $\gamma$ ); C, nuclear factor kappa-light-chain-enhancer of activated $B$ cells (NF- $\kappa B, p 65)$. The nuclear proteins were compared with Lamin-B1. Results are represented as mean \pm SD. * Represents a significant difference $(p<0.05)$ compared to control; \# shows a significant difference between the ACL-treated and pre-treatment groups. 
this increase could be a protective measure as Nrf2 downstream activity denotes enhancing antioxidant and antiapoptotic effects on tissues. However, this observed effect begs for further investigation. B, shows Western blotting analysis of PPAR $\gamma$ phosphorylated protein expression in each experimental group. Our result revealed that following pre-treatments with either LTN or SPN, PPAR $\gamma$ expressions increased significantly $(p<0.001)$ compared to control. This result, in addition, confirmed that LTN has the potential to enhance the activity of $\operatorname{PPAR} \gamma$. Moreover, pre-treatment with the combination of SPN and LTN also showed an increase in the PPARy expression compared to control. These results suggest a corresponding increase with $\mathrm{Nrf2}$ along with PPAR $\gamma$ expressions indicating diminished oxidative stress induction by ACL. In $\mathrm{C}$, we present protein expressions of $\mathrm{NF}-x \mathrm{~B}$ in the wake of $\mathrm{ACL}$ treatment and pre-treatments with LTN and SPN. As expected, ACL increased $(p<0.01)$ the expressions of $\mathrm{NF}-x \mathrm{~B}$ in the heart samples implying oxidative stress and inflammatory condition. We also found that only pre-treatment with LTN and the combination of SPN+LTN produced a significant $(p<0.05)$ decrease in $\mathrm{NF}-x \mathrm{~B}$ expression in heart samples.

The results of the Western blotting protein analysis are presented in Figure 6 (A-B). In A, we present the protein expressions of COX-2 in all experimental groups. It revealed that ACL significantly $(p<0.001)$ increased COX-2 expression compared to control. Pre-treatment with LTN, SPN, and their combination was found to decrease COX-2 expressions. This could suggest that ACL induction of oxidative stress might be multifactorial. Figure 6 B. shows the effects of treatment
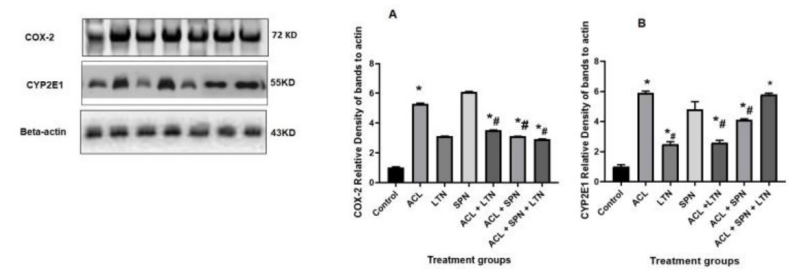

Figure 6: Effects of acrolein (ACL), losartan (LTN), and sulforaphane (SPN), and pretreatments with LTN, SPN, and SPN+LTN after 4 weeks on A, different bands of protein expressions, and relative density of beta-actin. A, cyclooxygenase-2 (COX-2) and B, cytochrome P450 2E1 (CYP2E1) protein expressions. Cytoplasmic protein extracts extracts were compared with Beta-actin. Results are represented as mean \pm SD. * Represents a significant difference $(p<0.05)$ compared to control; \# shows a significant difference between the ACL-treated and pre-treatment groups. with ACL and pre-treatments with LTN and SPN on protein expressions of CYP2E1. Our data show that ACL enhanced this expression considerably $(p<0.001)$ compared to control. In a similar fashion, both LTN and SPN significantly $(p<0.001)$ suppressed the expression of CYP2E1 compared to ACL treatment. Unexpectedly, we observed that the combined pre-treatment of SPN and LTN produced an increase in CYP2E1 expression compared to the ACL treatment, which we cannot explain.

\section{DISCUSSION}

Evidence from numerous studies reveals that cardiac tissue is very sensitive to the toxic effects of ACL., ${ }^{1,22}$ This is due to the ability of ACL to form complex protein adducts in the cardiac mitochondria, resulting in decreased energy utilization. ${ }^{22}$ In this study, we have shown that ACL-induced cardiomyopathy was produced via the activation of NF- $x \mathrm{~B}, \mathrm{COX}-2$, and CYP2E1 protein expressions. These signaling pathways have been reported to induce oxidative stress, inflammation and, in their wake, release proinflammatory mediators that induce oxidative stress $^{23-25}$ Our present report also demonstrated that these effects were attenuated by pre-treatment with either SPN or LTN and more so with their combination. We, therefore, suggest that the SPN+LTN combination suppressed the activities of pro-oxidative signaling pathways by stimulating the downstream protein expressions of Nrf2 and PPAR $\gamma$. According to documented evidence, SPN has been identified as a potent antioxidant and gene expression regulator, acting via the induction of $\mathrm{Nrf2}$ activity. ${ }^{26} \mathrm{In}$ contrast, LTN (an agonist of PPARy) is said to improve cardiac function by decreasing oxidative stress by stimulating the PPAR $\gamma$ signaling pathway, ${ }^{27,28}$ In terms of oxidative stress induced by ACL, our study showed that GSH levels were increased significantly. Our finding is likened to the report of Sthijins et al. ${ }^{7}$ and Uemura et al. ${ }^{29}$ indicating that ACL apparently induces an adaptive measure whereby GSH level is increased during oxidative stress conditions. They report that as the concentration of ACL in the cell rises, it elicits a consequent increase in GSH level within the cell. This observed effect might be due to the induction of several antioxidant signaling pathways as a self-protective measure. ${ }^{30}$ We report that pretreatment with either SPN or LTN suppressed the effect of ACL, indicating reduced oxidant activity. Our observation is complemented by similar reported findings, demonstrating the ability of SPN and LTN to reduce induced oxidative stress both in the heart and other tissues. ${ }^{31,32}$ However, the combination of SPN and 
LTN gave a profound reduction, suggesting that it has a better potential to attenuate ACL cardiomyopathy. In a similar fashion, ACL also caused a significant increase in catalase activity. We, therefore, suggest that this similar action resembles the increase of GSH level observed and is reminiscent of the same mechanism that might be at play here. It exemplifies dynamic cardioprotection towards ACL-induced cardiomyopathy. ${ }^{7}$ Compared to ACL treatment, combined pre-treatment with SPN and LTN produced a significant decrease in catalase activity to the same level as the control. However, our reported finding is contrary to previous studies conducted with cell lines, where ACL reportedly reduced catalase activity. ${ }^{31}$ Expectantly, in the present study, ACL increased cardiac lipid peroxidation, which was attenuated by SPN. Numerous studies have documented similar results in line with our own findings. ${ }^{31,33}$ Drug-induced lipid peroxidation has been linked to cardiac injury, including cardiomyopathy. ${ }^{34,35}$ Therefore, agents that will mitigate this aspect of oxidative stress product will ameliorate cardiac disease progression. We hereby recount again that the combination of SPN and LTN completely abolished the effect of ACL in generating lipid peroxidation, suggesting a better potential antioxidant profile.

CK-MB, a product of damaged myocyte is usually a sensitive marker of myocardial injury. ${ }^{36}$ From the foregoing, we present here an increased level of CK-MB in rats treated with ACL for 4 weeks, which is in line with previously documented studies. ${ }^{36,37}$ In addition, we also found a significant reduction in the levels of CK-MB amongst rats pre-treated with either SPN or LTN, and this finding is corroborated in the literature. ${ }^{38}$ Elevated levels of cardiac troponin have been described as an early marker for cardiomyopathy. ${ }^{39}$ Whereas $\mathrm{Li}$ et $a l^{40}$ after reporting the association of elevated cardiac troponin with cardiomyopathy, advocated for drugs that can modulate troponin for the treatment of cardiomyopathy, signifying the role of troponin in the disease progression. In like manner, cardiac troponin was also found to be elevated by ACL and attenuated by pretreatment with SPN and LTN, as were also documented by various workers. ${ }^{35,41}$ In addition, SPN+LTN pretreatment restored troponin levels as seen with the control. The result suggests that the combination could be a good candidate to stem the progression of heart injury to cardiomyopathy, as a report has linked increased cardiac troponin to apoptosis. ${ }^{42}$ The critical role that caspase 3 plays in apoptosis has been recognized as the primary factor in cell death. ${ }^{43}$ Numerous recent and past studies have shown that ACL induces apoptosis via the induction of the caspase 3 signaling pathway. ${ }^{36,44,45}$ Our data support these findings in that we also observed a significant increase in caspase 3 activity amongst ACLtreated rats. As confirmed by the studies of Ma et al. ${ }^{46}$ and Fernandos et $\mathrm{al} .{ }^{47}$ we also report that SPN attenuated the effect of ACL on caspase 3 activity. The implication, therefore, is the diminishing of apoptosis, consequent upon abolishment of attendant cardiomyopathy. The reversal of ACL cardiac damage by SPN has also been documented befor. ${ }^{38,48}$ This documented evidence confirms our findings, suggesting that combined treatment with SPN and LTN has shown a potential therapeutic agent for ACL-induced cardiomyopathy. The attenuation of cardiomyopathy-induced histopathological changes by SPN and LTN, both individually and in combination, reflects their ability to mitigate cardiac oxidative and cardiac injury biomarkers levels. Hence, we observe normal striation, normal monocyte nucleus, and diminished myocyte disarray in heart samples examined in these pre-treatment groups, compared to ACL.

The upregulation of Nrf2 expression came with downregulation of NF- $x \mathrm{~B}, \mathrm{COX}-2$, and CYP2E1 expressions, evidenced by attenuation of ACL-induced cardiomyopathy in this study by SPN. ${ }^{31,49,50}$ According to Hayes and Dinkova-Kostova, ${ }^{51} \mathrm{Nrf} 2$ allows adaptation by promoting the induction of antioxidant genes to maintain redox homeostasis. Hence, this could explain our observation of increased Nrf2 expressions. The overall effect is the modulation of oxidative stress and apoptosis involved in cardiomyopathy. ${ }^{47}$ On the other hand, evidence shows that activation of PPARy signaling suppresses inflammation induced by NF- $x \mathrm{~B}$ and COX-2 upregulation. ${ }^{28}$ Previous reports showed that ACL-induced toxicity reduced PPARy level with increased NF- $x \mathrm{~B}$ and COX-2 activities, in line with our present observation. ${ }^{52}$ In addition, PPARy down and upregulation can be affected by the actions of inducible CYP2E1.53,54 Therefore, the mechanism by which SPN and its combination with LTN mediate the antioxidant and antiapoptotic effects in drug-induced cardiomyopathy has been documented. ${ }^{31,55-58}$ However, according to a recent study, SPN can affect the activity of CYP2E1 in conditions of oxidative stress. ${ }^{50}$ An increase in CYP2E1 expression in the heart has been reported to induce oxidative stress and apoptosis, leading to cardiomyopathy. ${ }^{25}$ From the foregoing, our present report showed upregulation of $\mathrm{Nrf} 2$ and PPARy expressions by a combination of SPN and LTN, which seemly ameliorated the effect of ACL. Also consistent with our finding is that both Nrf2 and PPARy reduced troponin levels and decreased caspase 3 activities.$^{59,60}$ However, literature is silent as regards the mechanism by which SPN and LTN could potentiate 
their effect in the attenuation of ACL-induced cardiomyopathy. Therefore, in this study, we further evaluated their potential mechanism when combined. According to documented studies, Nrf2 and PPARy signaling pathways appear to be linked positively. ${ }^{13}$ Also reported is that PPARy agonists were found to induce the transcription of factor genes belonging to Nrf2, suggesting that PPARy can regulate the Nrf2 pathway. ${ }^{61}$ In addition, expressions of Nrf2 were suppressed in PPARy null mouse model. ${ }^{14}$ Therefore, it is suggested that PPARy and transcription factor Nrf2 could be acting synergistically to activate antioxidant factor genes. $^{62}$ From the present study, we suggest that simultaneous activation of these signaling pathways could portend a beneficial effect in conditions of cardiomyopathy. In the present study, we observed that proinflammatory mediators' expressions were all attenuated, with a consequent reduction in both troponin and caspase 3 activity. Hence, also suggesting that they might be acting in a synergistic fashion. From the foregoing, therefore, the effect of SPN+LTN combination on ACL-induced cardiomyopathy appears to have therapeutic potential and should be further investigated. Studies have shown that treatment with SPN improved cardiac function and remodelling by inhibiting inflammation and oxidative stress in a rabbit model of chronic heart failure. ${ }^{46,63} \mathrm{LTN}$ also has been documented and approved for patients with heart failure. ${ }^{64}$ The present study could potentially indicate that SPN, together with LTN, could potentially exert a cardiovascular protective effect by activating $\operatorname{PPAR} \gamma / \mathrm{Nrf} 2$ signaling. Hence, inducing the expressions of antioxidant enzymes that will reduce oxidative stress, ultimately improving cardiovascular complications and dysfunction in the clinical settings. Therefore, synergistic use in the clinical application should be exploited. . $2,49,65,66^{-1}$ One of the limitations of this study is that the authors did not observe the animals further to check if the induced cardiomyopathy is reversible. Secondly, we did not include mortality data.

\section{CONCLUSION}

For the first time, we report the combination of Nrf2 and PPARy agonists in the attenuation of ACLinduced cardiomyopathy. In this study, we explored the mechanisms by which SPN modulates other signaling pathways involved in cardioprotection via the activation of the Nrf2 pathway. We demonstrated that activation of Nrf2, which suppressed NF- $x$ B, COX-2, and CYP2E1 pathways, was upregulated by PPAR $\gamma$ that known to play a pivotal role in cardioprotection by reducing cardiac injury. LTN acted in a similar fashion, as was observed with SPN. However, the combination of SPN and LTN appeared to act synergistically to attenuate the effect of ACL-induced oxidative stress. These results suggest that the pharmacological activation of PPAR $\gamma$ interplays with Nrf2 to suppress the cardiomyopathy induced by ACL. Therefore, this may provide a better therapeutic benefit against cardiomyopathy induced by ACL.

\section{ACKNOWLEDGEMENT}

Authors hereby thank Deanship of Scientific Research, King Faisal University, Saudi Arabia for funding this research with a funded grant number: 160010. We also wish to acknowledge Mr. Tameem Alyahian, our Laboratory Supervisor, for his assistance.

\section{CONFLICT OF INTEREST}

The authors declare no conflict of interest.

\section{Institutional Review Board Statement}

The study was conducted according to the guidelines of the Declaration of Helsinki and approved by Research Ethics Committee (REC) of Deanship of Scientific Research, King Faisal University (approval No. KFUREC/2021-06-07).

\section{Author Contributions}

Conceptualization, P.M.E; Methodology, P.M.E.; H-I.M.I.; M.A.M.; I.A.A.; S.N.H. and E.A.A.; Formal analysis, P.M.E.; H-I.M.I. and E.A.A.; Writingoriginal draft preparation, P.M.E.; H-I.M.I. and E.A.A.; Writing_review and editing, P.M.E.; H-I.M.I.; M.A.M.; I.A.A.; S.N.H. and E.A.A.; Supervision, P.M.E.; H-I.M.I.; I.A.A. and E.A.A.; Project administration, P.M.E.; Funding acquisition, P.M.E. All authors have read and agreed to the published version of the manuscript.

\section{REFERENCES}

1. Wang GW, Guo Y, Vondriska TM, Zhang J, Zhang S, Tsai LL, et al. Acrolein consumption exacerbates myocardial ischemic injury and blocks nitric oxideinduced PKCepsilon signaling and cardioprotection. J Mol Cell Cardiol. 2008;44(6):1016-22. doi: 10.1016/j.yjmcc.2008.03.020, PMID 18468618.

2. Grootveld M, Percival BC, Leenders J, Wilson PB. Potential adverse public health effects afforded by the ingestion of dietary lipid oxidation product toxins: Significance of fried food sources. Nutrients. 2020;12(4):E974. doi: 10.3390/nu12040974, PMID 32244669.

3. Ismahil MA, Hamid T, Haberzettl P, Gu Y, Chandrasekar B, Srivastava S, et al. Chronic oral exposure to the aldehyde pollutant acrolein induces dilated cardiomyopathy. Am J Physiol Heart Circ Physiol. 2011;301(5):H2050-60. doi: 10.1152/ajpheart.00120.2011, PMID 21908791.

4. Lorenz DR, Misra V, Chettimada S, Uno H, Wang L, Blount BC, et al. Acrolein and other toxicant exposures in relation to cardiovascular disease among marijuana and tobacco smokers in a longitudinal cohort of HIV-positive and negative adults. E Clinical Medicine. 2021;31:100697. doi: 10.1016/j. eclinm.2020.100697. 
5. Pei Z, Meng R, Zhuang Z, Zhao Y, Liu F, Zhu MZ, et al. Cardiac peroxisome proliferator-activated receptor- $Y$ expression is modulated by oxidative stress in acutely infrasound-exposed cardiomyocytes. Cardiovasc Toxicol. 2013;13(4):307-15. doi: 10.1007/s12012-013-9211-5, PMID 23632742.

6. Conklin DJ, Barski OA, Lesgards JF, Juvan P, Rezen T, Rozman D, et al. Acrolein consumption induces systemic dyslipidemia and lipoprotein modification. Toxicol Appl Pharmacol. 2010;243(1):1-12. doi: 10.1016/j. taap.2009.12.010, PMID 20034506.

7. Sthijns MM, Randall MJ, Bast A, Haenen GR. Adaptation to acrolein through upregulating the protection by glutathione in human bronchial epithelial cells: the materialization of the hormesis concept. Biochem Biophys Res Commun. 2014;446(4):1029-34. doi: 10.1016/j.bbrc.2014.03.081, PMID 24667599.

8. Polvani S, Tarocchi M, Galli A. PPARy and oxidative stress: con $(\beta)$ catenating NRF2 and FOXO. PPAR Res. 2012;2012:641087. doi: 10.1155/2012/641087.

9. Lee TI, Kao YH, Chen YC, Pan NH, Lin YK, Chen YJ. Cardiac peroxisomeproliferator-activated receptor expression in hypertension co-existing with diabetes. Clin Sci (Lond). 2011;121(7):305-12. doi: 10.1042/CS20100529, PMID 21501116.

10. Nordgren KK, Wallace KB. Keap1 redox-dependent regulation of doxorubicininduced oxidative stress response in cardiac myoblasts. Toxicol Appl Pharmacol. 2014;274(1):107-16. doi: 10.1016/j.taap.2013.10.023, PMID 24211725.

11. Wilding JP. PPAR agonists for the treatment of cardiovascular disease in patients with diabetes. Diabetes Obes Metab. 2012;14(11):973-82. doi: 10.1111/j.1463-1326.2012.01601.x, PMID 22443197.

12. Son NH, Park TS, Yamashita H, Yokoyama M, Huggins LA, Okajima K, et al. Cardiomyocyte expression of PPARgamma leads to cardiac dysfunction in mice. J Clin Invest. 2007;117(10):2791-801. doi: 10.1172/JCI30335, PMID 17823655.

13. Cho HY, Gladwell W, Wang X, Chorley B, Bell D, Reddy SP, et al. Nrf2regulated PPAR \{gamma\} expression is critical to protection against acute lung injury in mice. Am J Respir Crit Care Med. 2010;182(2):170-82. doi: 10.1164/rccm.200907-1047OC, PMID 20224069.

14. Lee TW, Bai KJ, Lee TI, Chao TF, Kao YH, Chen YJ. PPARs modulate cardiac metabolism and mitochondrial function in diabetes. J Biomed Sci. 2017;24(1):5. doi: 10.1186/s12929-016-0309-5, PMID 28069019.

15. Yang SH, Li P, Yu LH, Li L, Long M, Liu MD, et al. Sulforaphane Protect Against Cadmium-Induced Oxidative Damage in mouse Leydigs cells by Activating Nrf2/ARE Signaling Pathway. Int J Mol Sci. 2019;20(3):E630. doi: 10.3390/ijms20030630, PMID 30717178.

16. Cho HY, Miller-DeGraff L, Blankenship-Paris T, Wang X, Bell DA, Lih F, et al. Sulforaphane enriched transcriptome of lung mitochondrial energy metabolism and provided pulmonary injury protection via Nrf2 in mice. Toxicol Appl Pharmacol. 2019;364:29-44. doi: 10.1016/j.taap.2018.12.004, PMID 30529165.

17. Fimognari C, Lenzi M, Sestili P, Turrini E, Ferruzzi L, Hrelia P, Cantelli-Forti G. Sulforaphane potentiates RNA damage induced by different xenobiotics. PLOS ONE. 2012;7(4):e35267. doi: 10.1371/journal.pone.0035267, PMID 22539965.

18. Negi G, Kumar A, Sharma SS. Nrf2 and NF-KB modulation by sulforaphane counteracts multiple manifestations of diabetic neuropathy in rats and high glucose-induced changes. Curr Neurovasc Res. 2011;8(4):294-304. doi: 10.2174/156720211798120972, PMID 22023613.

19. Diogo LN, Faustino IV, Afonso RA, Pereira SA, Monteiro EC, Santos AI. Voluntary oral administration of losartan in rats. J Am Assoc Lab Anim Sci. 2015;54(5):549-56. PMID 26424254.

20. Emeka PM, Rasool ST, Morsy MA, Islam MIH, Chohan MS. Protective effects of lutein against vancomycin-induced acute renal injury in mice via upregulation of peroxisome proliferator-activated receptor gamma/nuclear factor erythroid 2-related factor 2 and inhibition nuclear factor-kappaB/ caspase 3. Korean J Physiol Pharmacol. 2021;25(4):321-31. doi: 10.4196/ kjpp.2021.25.4.321, PMID 34187949.

21. Billingham ME, Mason JW, Bristow MR, Daniels JR. Anthracycline cardiomyopathy monitored by morphologic changes. Cancer Treat Rep. 1978;62(6):865-72. PMID 667860.

22. Luo J, Hill BG, Gu Y, Cai J, Srivastava S, Bhatnagar A, et al. Mechanisms of acrolein-induced myocardial dysfunction: implications for environmental and endogenous aldehyde exposure. Am J Physiol Heart Circ Physiol. 2007;293(6):H3673-84. doi: 10.1152/ajpheart.00284.2007, PMID 17921335.
23. Soleimani A, Rahmani F, Ferns GA, Ryzhikov M, Avan A, Hassanian SM. Role of the NF-KB signaling pathway in the pathogenesis of colorectal cancer. Gene. 2020;726:144132. doi: 10.1016/j.gene.2019.144132.

24. Park YS, Kim J, Misonou Y, Takamiya R, Takahashi M, Freeman MR, et al. Acrolein induces cyclooxygenase-2 and prostaglandin production in human umbilical vein endothelial cells: roles of p38 MAP kinase. Arterioscler Thromb Vasc Biol. 2007;27(6):1319-25. doi: 10.1161/ATVBAHA.106.132837, PMID 17363696.

25. Guan F, Yang X, Li J, Dong W, Zhang X, Liu N, et al. New molecular mechanism underlying Myc-mediated cytochrome P450 2E1 upregulation in apoptosis and energy metabolism in the myocardium. J Am Heart Assoc. 2019;8(1):e009871. doi: 10.1161/JAHA.118.009871, PMID 30563421.

26. De Souza CG, Sattler JA, de Assis AM, Rech A, Perry ML, Souza DO. Metabolic effects of sulforaphane oral treatment in streptozotocin-diabetic rats. J Med Food. 2012;15(9):795-801. doi: 10.1089/jmf.2012.0016, PMID 22925073.

27. Khaper N, Singal PK. Modulation of oxidative stress by a selective inhibition of angiotensin II type 1 receptors in MI rats. J Am Coll Cardiol. 2001;37(5):1461-6. doi: 10.1016/s0735-1097(01)01126-3, PMID 11300462.

28. An J, Nakajima T, Kuba K, Kimura A. Losartan inhibits LPS-induced inflammatory signaling through a PPARgamma-dependent mechanism in human THP-1 macrophages. Hypertens Res. 2010;33(8):831-5. doi: 10.1038/hr.2010.79, PMID 20505677.

29. Uemura T, Nakamura M, Sakamoto A, Suzuki T, Dohmae N, Terui Y, et al. Decrease in acrolein toxicity based on the decline of polyamine oxidases. Int J Biochem Cell Biol. 2016;79:151-7. doi: 10.1016/j.biocel.2016.08.039, PMID 27590852.

30. Kwak MK, Kensler TW, Casero RA. Induction of phase 2 enzymes by serum oxidized polyamines through activation of Nrf2: effect of the polyamine metabolite acrolein. Biochem Biophys Res Commun. 2003;305(3):662-70. doi: 10.1016/s0006-291x(03)00834-9, PMID 12763045.

31. Qin WS, Deng YH, Cui FC. Sulforaphane protects against acroleininduced oxidative stress and inflammatory responses: modulation of Nrf-2 and COX-2 expression. Arch Med Sci. 2016;12(4):871-80. doi: 10.5114/ aoms.2016.59919, PMID 27478470.

32. Lin $\mathrm{CH}$, Yang $\mathrm{H}$, Xue QL, Chuang YF, Roy CN, Abadir P, et al. Losartan improves measures of activity, inflammation, and oxidative stress in older mice. Exp Gerontol. 2014;58:174-8. doi: 10.1016/j.exger.2014.07.017, PMID 25077714.

33. Lee SE, Park YS. Role of lipid peroxidation-derived $\alpha, \beta$-unsaturated aldehydes in vascular dysfunction. Oxid Med Cell Longev. 2013;2013:629028. doi: 10.1155/2013/629028.

34. Venardos KM, Perkins A, Headrick J, Kaye DM. Myocardial ischemiareperfusion injury, antioxidant enzyme systems, and selenium: A review. Curr Med Chem. 2007;14(14):1539-49. doi: 10.2174/092986707780831078, PMID 17584062.

35. Singhal SS, Singh SP, Singhal P, Horne D, Singhal J, Awasthi S. Antioxidant role of glutathione S-transferases: 4-hydroxynonenal, a key molecule in stress-mediated signaling. Toxicol Appl Pharmacol. 2015;289(3):361-70. doi: 10.1016/j.taap.2015.10.006, PMID 26476300.

36. Taghiabadi E, Imenshahidi M, Abnous K, Mosafa F, Sankian M, Memar B, et al. Protective effect of silymarin against acrolein-induced cardiotoxicity in mice. Evid Based Complement Alternat Med. 2012;2012:352091. doi: 10.1155/2012/352091.

37. Aydın B, Atlı Şekeroğlu Z, Şekeroğlu V. Effects of whey protein and conjugated linoleic acid on acrolein-induced cardiac oxidative stress, mitochondrial dysfunction and dyslipidemia in rats. Biomed Pharmacother. 2018;107:901-7. doi: 10.1016/j.biopha.2018.08.081, PMID 30257402.

38. Song L, Srilakshmi M, Wu Y, Saleem TSM. Sulforaphane attenuates isoproterenol-induced myocardial injury in mice. BioMed Res Int. 2020;2020(9):3610285. doi: 10.1155/2020/3610285.

39. Stege NM, De Boer RA, Van den Berg MP, Silljé HHW. The time has come to explore plasma biomarkers in genetic cardiomyopathies. Int $\mathrm{J}$ Mol Sci. 2021;22(6):2955. doi: 10.3390/ijms22062955, PMID 33799487.

40. Li MX, Hwang PM. Structure and function of cardiac troponin C (TNNC1): implications for heart failure, cardiomyopathies, and troponin modulating drugs. Gene. 2015;571(2):153-66. doi: 10.1016/j.gene.2015.07.074, PMID 26232335 . 
41. Ryba DM, Li J, Cowan CL, Russell B, Wolska BM, Solaro RJ. Long-term biased $\beta$-arrestin signaling improves cardiac structure and function in dilated cardiomyopathy. Circulation. 2017;135(11):1056-70. doi: 10.1161/ CIRCULATIONAHA.116.024482, PMID 28104714.

42. Weil BR, Young RF, Shen X, Suzuki G, Qu J, Malhotra S, et al. Brief myocardial ischemia produces cardiac troponin I release and focal myocyte apoptosis in the absence of pathological infarction in swine. JACC Basic Transl Sci. 2017;2(2):105-14. doi: 10.1016/j.jacbts.2017.01.006, PMID 28979949.

43. Kern JC, Kehrer JP. Acrolein-induced cell death: A caspase-influenced decision between apoptosis and oncosis/necrosis. Chem Biol Interact. 2002;139(1):79-95. doi: 10.1016/s0009-2797(01)00295-2, PMID 11803030.

44. Roy J, Pallepati P, Bettaieb A, Tanel A, Averill-Bates DA. Acrolein induces a cellular stress response and triggers mitochondrial apoptosis in A549 cells. Chem Biol Interact. 2009;181(2):154-67. doi: 10.1016/j.cbi.2009.07.001, PMID 19596284.

45. Kitaguchi Y, Taraseviciene-Stewart L, Hanaoka M, Natarajan R, Kraskauskas D, Voelkel NF. Acrolein induces endoplasmic reticulum stress and causes airspace enlargement. PLOS ONE. 2012;7(5):e38038. doi: 10.1371/journal.pone.0038038, PMID 22675432.

46. Ma T, Zhu D, Chen D, Zhang Q, Dong H, Wu W, et al. Sulforaphane, a natural isothiocyanate compound, improves cardiac function and remodeling by inhibiting oxidative stress and inflammation in a rabbit model of chronic heart failure. Med Sci Monit. 2018;24:1473-83. doi: 10.12659/msm.906123, PMID 29527002.

47. Fernandes RO, Bonetto JH, Baregzay B, De Castro AL, Puukila S, Forsyth H, et al. Modulation of apoptosis by sulforaphane is associated with PGC-1 $\alpha$ stimulation and decreased oxidative stress in cardiac myoblasts. Mol Cell Biochem. 2015;401(1-2):61-70. doi: 10.1007/s11010-014-2292-z, PMID 25481685.

48. Bai Y, Wang X, Zhao S, Ma C, Cui J, Zheng Y. Sulforaphane protects against cardiovascular disease via Nrf2 activation. Oxid Med Cell Longev. 2015;2015:407580. doi: 10.1155/2015/407580.

49. Ruhee RT, Suzuki K. The integrative role of sulforaphane in preventing inflammation, oxidative stress and fatigue: a review of a potential protective phytochemical. Antioxidants. (Basel). 2020;9(6):E521. doi: 10.3390/ antiox9060521, PMID 32545803.

50. Zhou R, Lin J, Wu D. Sulforaphane induces Nrf2 and protects against CYP2E1-dependent binge alcohol-induced liver steatosis. Biochim Biophys Acta. 2014;1840(1):209-18. doi: 10.1016/j.bbagen.2013.09.018, PMID 24060752.

51. Hayes JD, Dinkova-Kostova AT. The Nrf2 regulatory network provides an interface between redox and intermediary metabolism. Trends Biochem Sci. 2014;39(4):199-218. doi: 10.1016/j.tibs.2014.02.002, PMID 24647116.

52. Stokes PE, Sikes CR. The hypothalamic-pituitary-adrenocortical axis in major depression. Neurol Clin. 1988;6(1):1-19. doi: 10.1016/S07338619(18)30881-8, PMID 2837631.

53. Chen J, Yang $\mathrm{H}$, Sheng Z. Ellagic acid activated PPAR signaling pathway to protect ileums against castor oil-induced diarrhea in mice: application of transcriptome analysis in drug screening. Front Pharmacol. 2019;10:1681. doi: 10.3389/fphar.2019.01681, PMID 32082169.

54. Chhimwal J, Sharma S, Kulurkar P, Patial V. Crocin attenuates CCl4induced liver fibrosis via PPAR-y mediated modulation of inflammation and fibrogenesis in rats. Hum Exp Toxicol. 2020;39(12):1639-49. doi: 10.1177/0960327120937048, PMID 32633567.

55. Corssac GB, Campos-Carraro C, Hickmann A, da Rosa Araujo AS, Fernandes RO, Belló-Klein A. Sulforaphane effects on oxidative stress parameters in culture of adult cardiomyocytes. Biomed Pharmacother. 2018;104:165-71. doi: 10.1016/j.biopha.2018.05.031, PMID 29772437.

56. Kubo E, Chhunchha B, Singh P, Sasaki H, Singh DP. Sulforaphane reactivates cellular antioxidant defense by inducing Nrf2/ARE/Prdx6 activity during aging and oxidative stress. Sci Rep. 2017;7(1):14130. doi: 10.1038/ s41598-017-14520-8. PMID 29074861.

57. Koh EJ, Yoon SJ, Lee SM. Losartan protects liver against ischaemia/ reperfusion injury through PPAR-y activation and receptor for advanced glycation end-products down-regulation. Br J Pharmacol. 2013;169(6):1404-16. doi: 10.1111/bph.12229, PMID 23647130.

58. Fried ND, Morris TM, Whitehead A, Lazartigues E, Yue X, Gardner JD. Angiotensin II type 1 receptor mediates pulmonary hypertension and right ventricular remodeling induced by inhaled nicotine. Am J Physiol Heart Circ Physiol. 2021;320(4):H1526-34. doi: 10.1152/ajpheart.00883.2020, PMID 33577434.

59. He F, Doucet JA, Stephens JM. Caspase-mediated degradation of PPARgamma proteins in adipocytes. Obesity (Silver Spring). 2008;16(8):1735-41. doi: 10.1038/oby.2008.269, PMID 18497737.

60. Bonay M, Roux AL, Floquet J, Retory $\mathrm{Y}$, Herrmann JL, Lofaso F, et al. Caspase-independent apoptosis in infected macrophages triggered by sulforaphane via Nrf2/p38 signaling pathways. Cell Death Discov. 2015;1:15022. doi: 10.1038/cddiscovery.2015.22, PMID 27551455.

61. Chorley BN, Campbell MR, Wang X, Karaca M, Sambandan D, Bangura F, et al. Identification of novel NRF2-regulated genes by ChIP-Seq: influence on retinoid X receptor alpha. Nucleic Acids Res. 2012;40(15):7416-29. doi: 10.1093/nar/gks409, PMID 22581777.

62. Kvandová $M$, Majzúnová $M$, Dovinová I. The role of PPARgamma in cardiovascular diseases. Physiol Res. 2016;65(Suppl 3):S343-63. doi: 10.33549/physiolres.933439, PMID 27775420.

63. Bai Y, Chen Q, Sun YP, Wang X, Lv L, Zhang LP, et al. Sulforaphane protection against the development of doxorubicin-induced chronic heart failure is associated with Nrf2 Upregulation. Cardiovasc Ther. 2017;35(5):e12277. doi: 10.1111/1755-5922.12277, PMID 28636290.

64. Singh KD, Karnik SS. Angiotensin Type 1 receptor blockers in heart failure. Curr Drug Targets. 2020;21(2):125-31. doi: 10.2174/1389450120666190821 152000, PMID 31433752.

65. Wu L, Noyan Ashraf MH, Facci M, Wang R, Paterson PG, Ferrie A, Juurlink $\mathrm{BH}$. Dietary approach to attenuate oxidative stress, hypertension, and inflammation in the cardiovascular system. Proc Natl Acad Sci U S A. 2004;101(18):7094-9. doi: 10.1073/pnas.0402004101, PMID 15103025.

66. Kayabasi H, Yilmaz Z, Sit D, Kadiroglu AK, Yilmaz E. The effects of losartan on oxidative stress and inflammation in non-diabetic patients undergoing chronic hemodialysis. Eur Rev Med Pharmacol Sci. 2013;17(2):235-42. PMID 23377814. 
PICTORIAL ABSTRACT

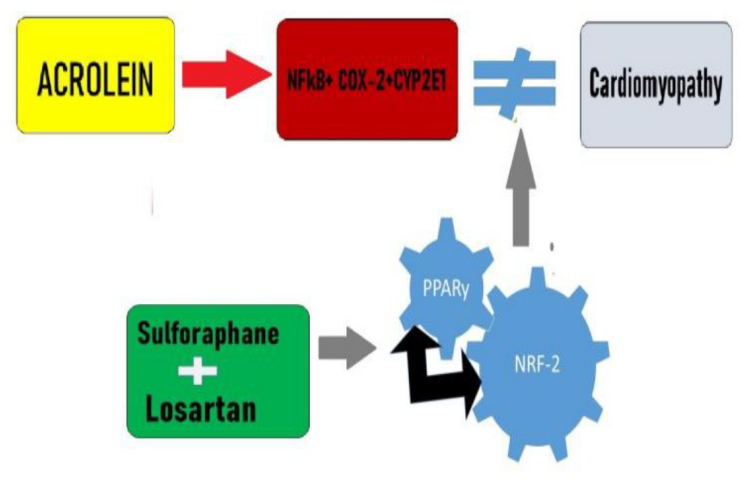

\section{SUMMARY}

The paper addresses the problem of acrolein that might be ingested unintentionally or by accident. Cardiomyopathy from acroelin has been a health concern and treatment is still elusive. In this study, we modulated the effects of ACL induced cardiomyopathy by enhancing the expressions of Nrf2 via PPARy. Hence will attenuated the expressions of nuclear factor kappa-light-chain-enhancer of activated B cells (NF- $x \mathrm{~B})$, cyclooxygenase-2 (COX-2), and cytochrome P450 2E1 (CYP2E1). The expressions of PPARy was increased by administration of LTN, a PPARy agonist, which enhanced the effect of SPN, an Nrf2 activator. The results here suggests a potential treatment option that can be beneficial to this ubiquitous compound, acrolein. Therefore, the report presents a combination of agents that appear to work synergistically in mitigating acrolein induced cardiomyopathy.

\section{About Authors}

Promise Madu Emeka holds a PhD in Pharmacology/Toxicology and is currently working as an Associate Professor at Department of Pharmaceutical Sciences College of Clinical Pharmacy, King Faisal University, AlAhsa, Saudi Arabia.

Hairul-Islam Mohamed lbrahim holds a PhD in Biotechnology and Immunology is currently working as an Assistant Professor at in Department of Biology, College of Science, King Faisal University, Al-Ahsa Saudi Arabia.

Mohamed Aly. Morsy holds a PhD in Pharmacology and is currently working as a Professor at Department of Pharmaceutical Sciences College of Clinical Pharmacy, King Faisal University, Al-Ahsa, Saudi Arabia.

Ibrahim Abdulrahman Alhaider holds a PhD in Pharmacology and was an Associate Professor at Department of Pharmaceutical Sciences College of Clinical Pharmacy, King Faisal University, but currently Assistant CEO for Research and Laboratories, Saudi Food and Drug Authority, Riyadh Saudi Arabia.

Snawar Hussain holds a PhD in Biochemistry and Molecular Biology and is currently working as an Assistant Professor at Department of Biomedical Sciences, College of Clinical Pharmacy King Faisal University, Al-Ahsa Saudi Arabia.

Emad Abdelaziz Ahmed holds a PhD in Molecular Physiology, with interest in cytogenetics and meiosis. Presently working as a Professor in Department of Biology, College of Science, King Faisal University, Al-Ahsa, Saudi Arabia.

Cite this article: Emeka PM, Ibrahim HM, Morsy MA, Alhaider IA, Hussain SN, Ahmed EA. Attenuation of Cardiomyopathy Induced in Sub-Chronic Exposure of Acrolein by Sulforaphane via Indirect PPARy Expression Promoter. Indian J of Pharmaceutical Education and Research. 2021;55(4):1048-59. 